\title{
A Rare Neck Mass: Extraskeletal Ewing's Sarcoma
}

\section{Banu Atalay Erdogan, Ozgur Sekercan, Fatih Bora, Gokhan Altin, Mustafa Paksoy, Sevim Baykal Koca}

\begin{abstract}
Extraskeletal Ewing's sarcoma (ESS) is rarely found in the head and neck region. This tumor is a rare soft tissue tumor morphologically similar to the Ewing's sarcoma orginating from bone. We report a case of extraskeletal Ewing's sarcoma of the posterior neck region in a 24-year-old woman. Magnetic resonance imaging examination showed a large soft tissue mass in the left paraspinal region. The final diagnosis was extraskeletal Ewing's sarcoma after excision of the mass. The patient showed good response to chemotherapy and radiotherapy.
\end{abstract}

Keywords: Extraskeletal Ewing's sarcoma, Neck, Treatment.

How to cite this article: Erdogan BA, Sekercan O, Bora F, Altin G, Paksoy M, Koca SB. A Rare Neck Mass: Extraskeletal Ewing's Sarcoma. Int J Head and Neck Surg 2012;3(3):165-167.

\section{Source of support: Nil}

Conflict of interest: None declared

\section{INTRODUCTION}

Extraskeletal Ewing's sarcoma (EES) is an uncommon, rapidly growing, round-cell malignancy of uncharacterized mesenchymal cell origin. Tefft et al first described EES in 1969 to be histologically similar to primary Ewing's sarcoma of bone. ${ }^{1}$ EES is a rare soft tissue neoplasms, that can develop in the soft tissues at any location. Its occurrence in head and neck as a primary tumor is very unusual. It occurs predominantly in adolescents and young adults between the ages of 10 and 30 years and it follows an aggressive course with a high rate of recurrence. Distant metastases are also common in EES. We present a case of EES as a neck mass in the left posterior cervical region.

\section{CASE REPORT}

A 24-year-old woman was referred to our Otolaryngology, Head and Neck Department with a rapidly enlarging painful left neck swelling. Physical examination showed a $3 \times 4 \mathrm{~cm}$ firm, mildly tender, mobile mass in the left posterior servical region (Fig. 1). There was no other abnormality in the head and neck. Head and neck magnetic resonance imaging (MRI) examination showed a well-defined, fusiform-shaped soft tissue tumor, measuring about $45 \times 28 \times 25 \mathrm{~mm}$, that was located in the left posterior paraspinal region (Figs $2 \mathrm{~A}$ and B). Fine needle aspiration cytology was not diagnostic. The patient underwent an operation which was excision of the tumor. Macroscopically, the tumor was mucoid cystic mass with a dark liquid. Histopathological examination showed features consistent with small round cell tumor. The tumor demonstrated high cellularity composed of small, round uniform cells with oval nuclei and scanty cytoplasm. The cytoplasmic organelles were poorly developed. Immunohistochemistry showed immunoreactivity to MIC 2 protein (CD99) and vimentin and nonreactivity to cytokeratin, actin, neuron-specific enolase and leukocyte common antigen. Thus histological and immunohistochemical findings were confirmatory for EES (Figs 3A and B). Whole body PET scanning revealed no other suspicious lesions. The patient was treated with multiagent chemotherapy and concurrent radiotherapy. The patient is tumor free with clinical or radiological evidence of tumor recurrence 36 months after treatment.

\section{DISCUSSION}

EES is a rare malignant neoplasm of uncharacterized mesenchymal cell origin. EES usually arises in the soft tissues of the trunk, paravertebral region, intercostal area, lower extremities and pelvis. ${ }^{2,3}$ The head and neck region is an unusual primary site for this type of tumor and, according to Chao et al, there were only five out of 118 cases of EES located in the head and neck region in which the nose, eyelid, nasopharynx, parotid gland, scalp and cervical region have been described. ${ }^{4,5-12}$ Only one case has been reported in parapharyngeal space. ${ }^{13}$ There is no sex predilection for this condition prior to adolescence; however, the number of males affected is slightly higher than the number of females after adolescence and may account for the increased incidence in males. Allam et $\mathrm{al}^{14}$ has reported a male to female ratio of 2.4:1 in a series of 24 patients.

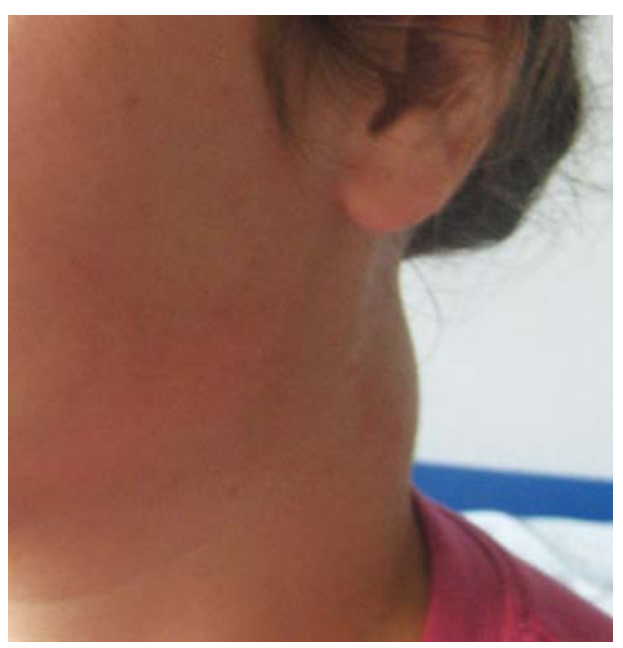

Fig. 1: Patient's neck examination showing a $3 \times 4 \mathrm{~cm}$ mass in the left posterior region 


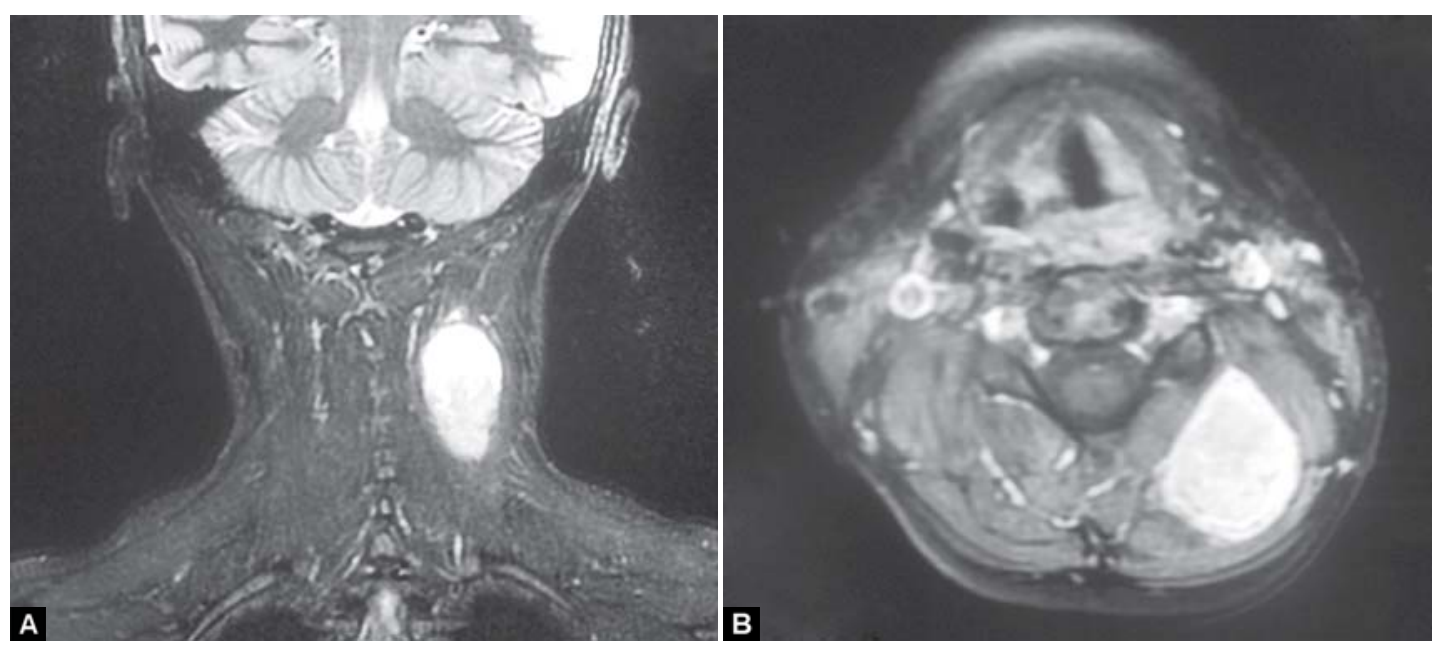

Figs 2A and B: T2-W axial image showed hyperintense signal in the mass. After intravenous gadolinium injection, heterogeneous contrast enhancement is detected

Young adolescents and adults between the ages of 10 and 30 years are predominantly affected, however, cases have been documented in patients between 14 months and

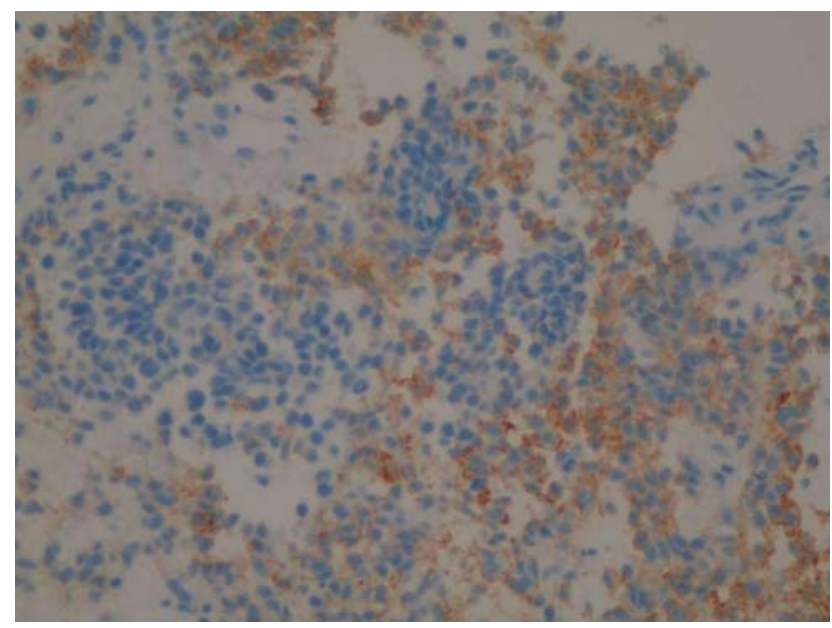

Fig. 3A: Immunohistochemical study of the EES showing diffuse positive staining for CD99 (PAS ×400)

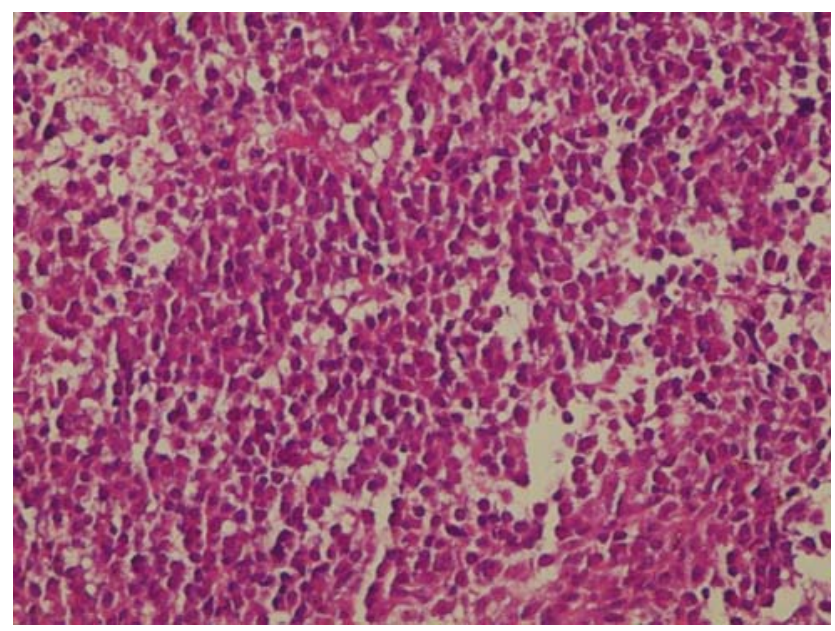

Fig. 3B: Histology showing the EES consisting of sheets and intersecting cords of intermediate-sized round cells with vacuolated oval nuclei, small nucleoli $(H \& E \times 200)$
63 years of age. ${ }^{2,3}$ Around $75 \%$ patients present with rapidly growing painless mass, 30\% patients exhibiting distant metastasis at the time of diagnosis. Clinically, patients with EES usually present with a painless, rapidly growing mass but tumors arising elsewhere may be painful. Our patient presented with a rapidly growing mass in the left side of neck. It usually has a pseudo capsule that makes it appear well circumscribed. Computed tomographic (CT) scan may show a low attenuation mass with heterogeneous enhancement with contrast administration. MRI findings include signal isointense to muscle on T1-W images and hyperintense on $\mathrm{T} 2-\mathrm{W}$ images with heterogeneous enhancement after gadolinium administration. ${ }^{15}$ Spontaneous tumor hemorrhage, adjacent bone destruction and regional metastatic adenopathy have been demonstrated in some cases using MRI or CT, but no evidence of tumoral calcification prior to treatment has been reported. ${ }^{4,6,7,10,15}$ In accordance with the previous description, the tumor from our patient appeared to be well circumscribed, inhomogeneous and showed no calcification on CT.

The histological image of EES is a small, blue, round cell with a scanty cytoplasm and it is often confused with other small round cell tumors. Angerwall and Enzinger first reported the pathological features and behavior of ESS, which are round or oval cell sarcoma of soft tissue and are histologically indistinguishable from ES of bone. ${ }^{2}$ Both ES and PNET show varying degree of neuroectodermal differentiation. EES is histologically similar to osseous ES and is often confused with other round cell tumors but recent advances in immunohistochemistry have helped in diagnosis of ESS. This family of tumors shares common cytogenetic and molecular changes which involve of Ewing's sarcoma gene on chromosome 22 (22q12) on to a number of other genes like fli-1 on chromosome 11(11q24) in 90\% of cases and ERG on chromosome 21 (21q22). ${ }^{7}$ The tumors also 
share expression of glycoprotein surface antigen p30/32 (MIC2) CD99, a cell membrane protein of unknown function. ${ }^{16}$ EES is a tumor sensitive to multimodality treatment. Early awareness and wide resection followed by chemotherapy and radiotherapy might improve the longterm survival of patients with extraskeletal Ewing's sarcoma. The rate of survival in EES has improved from 8\% to nearly $65 \%$ in patients with extremity lesions and to $83 \%$ in patients with truncal lesions, and the prognosis is now approaching that of skeletal Ewing's tumors. ${ }^{10}$

Five-year survival rates of EES according to various reports have been anywhere between 38 and 67\%. Although the prognosis for this tumor is poor, an early and adequate surgical resection followed by adjunctive chemotherapy and radiotherapy for microscopically positive surgical margins improve the survival rate. ${ }^{3,17}$ Careful preoperative planning in patients without metastatic disease for tumor-free wide surgical margins has a favorable prognosis. ${ }^{3}$ The aims of head and neck surgery are to improve or preserve the quality of life, achieve locoregional control and long-term survival.

\section{REFERENCES}

1. Tefft M, Vawter GF, Mitus A. Paravertebral 'round cell' tumours in children. Radiology 1969;92(7):1501-09.

2. Angervall L, Enzinger FM. Extraskeletal neoplasm resembling Ewing's sarcoma. Cancer 1975;36(1):240-51.

3. Rud NP, Reiman HM, Pritchard DJ, Frassica FJ, Smithson WA. Extraosseous Ewing's sarcoma: A study of 42 cases. Cancer 1989;64(7):1548-53.

4. Chao TK, Chang YL, Sheen TS. Extraskeletal Ewing's sarcoma of the scalp. J Laryngol Otol 2000;114(1):73-75.

5. Pontius KI, Sebek BA. Extraskeletal Ewing's sarcoma arising in the nasal fossa. Light- and electron-microscopic observations. Am J Clin Pathol 1981;75(3):410-15.

6. Reynoso VM, Carrillo J, Mora MA, Ridaura C. Ewing's sarcoma: Report of a case in the nasopharynx. Ear Nose Throat J 1984;63(9):459-62.

7. Lane S, Ironside JW. Extra-skeletal Ewing's sarcoma of the nasal fossa. J Laryngol Otol 1990;104(7)570-73.

8. Lim TC, Tan WT, Lee YS. Congenital extraskeletal Ewing's sarcoma of the face: A case report. Head Neck 1994;16(1): 75-78.

9. Zachariades N, Koumoura F, Liapi-Avgeri G, Bouropoulou V. Extraskeletal Ewing's sarcoma of the parotid region: A case report with the detection of the tumour's immunophenotypical characteristics. Br J Oral Maxillofac Surg 1994;32(5):328-31.

10. Kennedy JG, Eustace S, Caulfield R, Fennelly DJ, Hurson B, O’Rourke KS. Extraskeletal Ewing's sarcoma: A case report and review of the literature. Spine 2000;25(15):1996-99.
11. Boor A, Jurkovic I, Friedmann I, Plank L, Kocan P. Extraskeletal Ewing's sarcoma of the nose. J Laryngol Otol 2001;115(1): 74-76.

12. Shin JH, Lee HK, Rhim SC, Cho KJ, Choi CG, Suh DC. Spinal epidural extraskeletal Ewing's sarcoma: MR findings in two cases. AJNR Am J Neuroradiol 2001;22(4):795-98.

13. Ng SH, Ko SF, Cheung YC, Wong HF, Jung SM. Extraskeletal Ewing's sarcoma of the parapharygeal space. Br J Radiol 2004;77(924):1046-49.

14. Allam A, El-Husseiny G, Khafaga Y, Kandil A, Gray A, Ezzat A, et al. Ewing's sarcoma of the head and neck: A retrospective analysis of 24 cases. Sarcoma 1999;3(1):11-15.

15. O’Keeffe F, Lorigan JG, Wallace S. Radiological features of extraskeletal Ewing's sarcoma. Br J Radiol 1990; 63(750): 456-60.

16. de Alava E, Kawai A, Healey JH, Fligman I, Meyers PA, Huvos Ag, et al. EWS-FLI1 fusion transcript structure is an independent determinant of prognosis in Ewing's sarcoma. J Clin Oncol 1998 Aug;16(8):2895.

17. Raney RB, Asmar L, Newton WA Jr, et al. Ewing's sarcoma of soft tissues in childhood: A report from the intergroup rhabdomyosarcoma study 1972 to 1991 . J Clin Oncol 1997;15(2):574-82.

\section{ABOUT THE AUTHORS}

\section{Banu Atalay Erdogan (Corresponding Author)}

Department of Otolaryngology, Dr Lutfi Kirdar Kartal Education and Research Hospital, Istanbul, Turkey, e-mail: banuatalay81@gmail.com

\section{Ozgur Sekercan}

Department of Otolaryngology, Istanbul Education and Research Hospital, Istanbul, Turkey

\section{Fatih Bora}

Professor, Department of Otolaryngology, Kafkas University, Kars Turkey

\section{Gokhan Altin}

Department of Otolaryngology, Dr Lutfi Kirdar Kartal Education and Research Hospital, Istanbul, Turkey

\section{Mustafa Paksoy}

Department of Otolaryngology, Dr Lutfi Kirdar Kartal Education and Research Hospital, Istanbul, Turkey

\section{Sevim Baykal Koca}

Department of Pathology, Istanbul Education and Research Hospital Istanbul, Turkey 